\title{
Energy release associated with a first-order phase transition in a rotating neutron star core
}

\author{
J. L. Zdunik ${ }^{1}$, M. Bejger ${ }^{1,2}$, P. Haensel ${ }^{1}$, and E. Gourgoulhon ${ }^{2}$ \\ 1 N. Copernicus Astronomical Center, Polish Academy of Sciences, Bartycka 18, 00-716 Warszawa, Poland \\ e-mail: [jlz; bejger; haensel] @acamk.edu.pl \\ 2 LUTH, UMR 8102 du CNRS, Observatoire de Paris, 92195 Meudon Cedex, France \\ e-mail: Eric.Gourgoulhon@obspm.fr
}

Received 6 October 2006 / Accepted 2 January 2007

\begin{abstract}
Aims. We calculate energy release associated with a first order phase transition at the center of a rotating neutron star. This quantity is equal to the difference in mass-energies between the initial normal phase configuration and the final configuration containing a superdense matter core, with total baryon number and angular momentum kept constant.

Methods. The calculations of the energy release are based on precise numerical 2D calculations, in which both the polytropic equations of state (EOS) as well as realistic EOS of the normal phase are used. Presented results are obtained for a broad range of metastability of initial configuration and size of the new superdense phase core in the final configuration. When the equatorial radius of the dense core of the superdense phase is much smaller than the stellar equatorial radius, analytical expressions for the energy release are obtained.

Results. For a fixed "overpressure", $\delta \bar{P}$, defined as the relative excess of central pressure of a collapsing metastable star over the pressure of the equilibrium first-order phase transition, the energy release $\Delta E$ remarkably does not depend on the stellar angular momentum and coincides with that for nonrotating stars with the same $\delta \bar{P}$. The energy release is proportional to $(\delta \bar{P})^{2.5}$ for small $\delta \bar{P}$, when sufficiently precise brute force 2D numerical calculations are not possible. At higher $\delta \bar{P}$, results of 1D calculations of $\Delta E(\delta \bar{P})$ for non-rotating stars are shown to reproduce, with very high precision, the exact 2D results for rotating stars.
\end{abstract}

Key words. dense matter - equation of state - stars: neutron - stars: rotation

\section{Introduction}

One of the intriguing predictions of some theories of dense matter in neutron-star cores is a phase transition into an "exotic" state (i.e., not observed in the laboratory). Theoretical predictions include boson condensation of pions and kaons, and deconfinement of quarks (see, e.g., Glendenning 2000; Weber 1999).

First-order phase transitions accompanied by discontinuities in the thermodynamical potential densities, are the most interesting as far as the structure and dynamics of neutron stars are concerned. In the simplest case, one considers states consisting of one pure phase. Because of the high degeneracy of matter constituents, effects of temperature are neglected. In thermodynamic equilibrium, the phase transition occurs at a well defined pressure $P_{0}$, and is accompanied by a density jump at the phase interface.

A first-order phase transition allows for a metastability of the pure "normal" (lower density) phase at $P>P_{0}$. Consequently, a metastable core could form during neutron-star evolution in which central pressure increases, due to accretion or spin-down. Then, nucleation of the exotic (higher density) phase implies formation of a core of the exotic phase and is accompanied by a core-quake and energy release. A theory that enables one to calculate the changes in stellar parameters implied by a first-order phase transition in a non-rotating neutron star was developed by Haensel et al. (1986) and Zdunik et al. (1987) (an earlier Newtonian theory was presented by Schaeffer et al. 1983, see also Diaz Alonso 1983). The energy released in a corequake was shown to depend strongly on the size of the dense phase core, the leading term being proportional to the fifth power of this core radius.

In the present paper we calculate the energy release due to a phase transition in a rotating neutron star. Our theory is based on $2 \mathrm{D}$ simulations and is much closer to reality than the 1D theory developed in Haensel et al. (1986) and Zdunik et al. (1987). In the real world, evolutionary processes that lead to the increase of the central density in neutron star (accretion, slowingdown), as well as the collapse itself, all occur in a rotating star. Of course, the 2D calculations are incomparably more difficult than the 1D ones. However, as we show in the present paper, when suitably parametrized, the energy released during a corequake depends only on the excess of the central pressure of the metastable configuration over $P_{0}$, being to a good approximation independent of the angular momentum of the collapsing star.

In the present paper we restrict ourselves to the case when the radius of the core of the new phase is much smaller than the stellar radius, and the typical energy release will be $\sim 10^{50} \mathrm{erg}$. This is to be contrasted with a strong phase transition, considered in relation to long gamma-ray bursts at cosmological distances, where the core radius is a sizable fraction of the stellar radius and energy release $\sim 10^{52}-10^{53}$ erg (see, e.g., Berezhiani et al. 2003).

The paper is organized in the following way. In Sect. 2 we introduce notations and describe general properties of the firstorder phase transitions in the stellar core with particular emphasis on the metastability and instability of neutron star cores. 
Analytic considerations, concerning the response of a star to a first-order phase transition at its center, and in particular, the calculation within the linear response approximation of the energy release associated with such a transition, are presented in Sect. 4. Analytic models of the EOSs with first-order phase transitions, allowing for very precise $2 \mathrm{D}$ calculations, are considered in Sect. 3.1, where we derive generic properties of the energy release due to a first order phase transition at the center of a rotating star. In Sect. 3.2 we present our results obtained for a realistic EOS of normal phase, and we confirm remarkable properties of the energy-overpressure relation, obtained in the previous section. In Sect. 5 we present practical formulae suitable for the calculation of energy release associated with a first order phase transition at the center of a rotating neutron star. Finally, Sect. 6 contains discussion of our results and several examples of the application of our formula for the energy release.

\section{EOS with a first order phase transition}

Let us consider a general case of a first-order phase transition between the N (normal) and S (superdense) phases of dense matter. At densities under consideration, all constituents of matter are strongly degenerate, and the temperature dependence of pressure and energy density can be neglected. At a given baryon density, $n_{\mathrm{b}}$, the energy density of the N-phase of matter (including rest energies of particles that are matter constituents) is $\mathcal{E}_{\mathrm{N}}\left(n_{\mathrm{b}}\right)$ and pressure $P_{\mathrm{N}}\left(n_{\mathrm{b}}\right)$. The baryon chemical potential = enthalpy per baryon in the $\mathrm{N}$ phase is $\mu_{\mathrm{N}}=\left(P_{\mathrm{N}}+\mathcal{E}_{\mathrm{N}}\right) / n_{\mathrm{b}}$. Similarly, one can calculate thermodynamic quantities for the $\mathrm{S}$-phase.

The local pressure $P$ is a proper thermodynamic variable, continuous and monotonous in the stellar interior. The equilibrium state of the matter at a given $P$ is realized at the minimum of enthalpy per baryon. For $P<P_{0}$, this minimum is realized by the $\mathrm{N}$ phase, and for $P>P_{0}-$ by the $\mathrm{S}$ one. The value of $P_{0}$ is obtained from the crossing condition $\mu_{\mathrm{N}}(P)=\mu_{\mathrm{S}}(P)$, which also yields the values of the matter densities, $\rho_{\mathrm{N}}$ and $\rho_{\mathrm{S}}$, and the corresponding baryon densities, $n_{\mathrm{N}}$ and $n_{\mathrm{S}}$, at the N-S phase coexistence interface. These parameters are obtained assuming thermodynamic equilibrium. A schematic plot of the EOS of matter with a first order phase transition $\mathrm{N}-\mathrm{S}$, in the vicinity of the phase transition point, is plotted in Fig. 1.

The solid segment of the N-phase curve, in Fig. 1, corresponds to the stable $\mathrm{N}$-phase state. For pressure above $P_{0}$, the $\mathrm{N}$ phase becomes metastable with respect to the conversion into the $S$ phase. The $S$ phase can appear through the nucleation process - a spontaneous formation of S-phase droplets. However, an energy barrier resulting from the surface tension at the N-S interface delays the nucleation for a time identified with a lifetime of the metastable state $\tau_{\text {nucl }}$. The value of $\tau_{\text {nucl }}$ decreases sharply with $P>P_{0}$, and drops to zero at some $P_{\text {crit }}$, where the energy barrier separating the $\mathrm{S}$-state from the $\mathrm{N}$-state vanishes. For $P>P_{\text {crit }}$ the $\mathrm{N}$ phase is simply unstable and converts with no delay into the $\mathrm{S}$ phase.

Consider a neutron star built of matter in the $\mathrm{N}$ phase. Its central pressure $P_{\mathrm{c}}$ increases during spin-down or accretion. A quasistatic compression of the $\mathrm{N}$-phase core moves matter into a metastable state with $P>P_{0}$. A metastable core of the $\mathrm{N}$ phase is bounded by a surface with $P=P_{0}$. As soon as the central compression timescale of metastable core $\tau_{\text {comp }}=P_{\mathrm{c}} / \dot{P}_{\mathrm{c}}$ becomes equal to $\tau_{\text {nucl }}$, droplets of the $S$ phase appear at the star center. This happens at central pressure $P=P_{\text {nucl }}<P_{\text {crit }}$. The $\mathrm{S}$-phase droplets introduce a pressure deficit, destabilize the metastable core and consequently, the whole star. The S-phase core grows, and the process of the $\mathrm{N} \longrightarrow \mathrm{S}$ phase transition proceeds until the

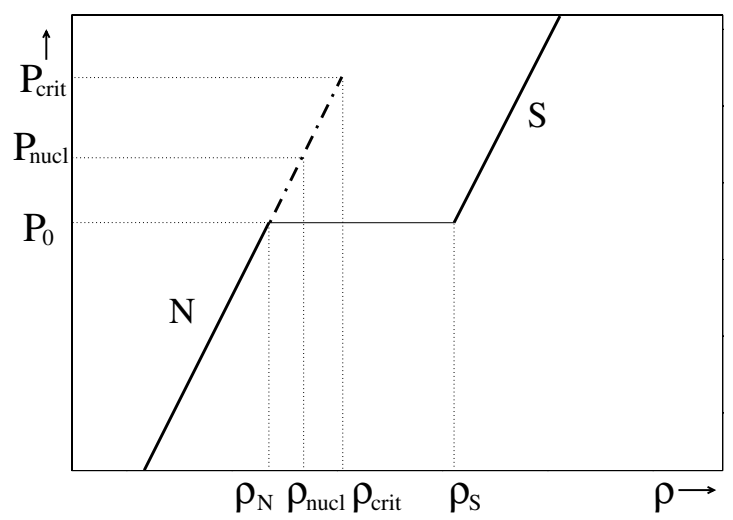

Fig. 1. A schematic representation, in the $\rho-P$ plane, of an EOS with a first order phase transition. Solid segments: stable $\mathrm{N}$ and $\mathrm{S}$ phase (in thermodynamic equilibrium). Dash-dot segment: metastable $\mathrm{N}$ phase. The $\mathrm{S}$ phase nucleates at $P_{\text {nucl }}$, which depends on the temperature and the compression rate. At $P_{\text {crit }}$ nucleation of the $\mathrm{S}$ phase is instantaneous, because the energy barrier, separating the $\mathrm{N}$ phase from the $\mathrm{S}$ phase, vanishes.

final hydrostatic equilibrium state is reached. In the final state, a central core of the S-phase is bounded by an N-S coexistence surface of constant pressure $P_{0}$ on which the matter density undergoes a jump from $\rho_{\mathrm{N}}$ on the outer $\mathrm{N}$-phase side to $\rho_{\mathrm{s}}$ on the inner S-phase side.

In the present paper we restrict ourselves to the case of the density jump satisfying $\rho_{\mathrm{S}}<\frac{3}{2}\left(\rho_{\mathrm{N}}+P_{0} / c^{2}\right)$. Therefore, stellar configurations with a core of the $S$ phase of arbitrarily small radius are stable (Seidov 1971; see also Kaempfer 1981; and Zdunik et al. 1987). The case of a strong first order phase transition with $\rho_{\mathrm{S}}>\frac{3}{2}\left(\rho_{\mathrm{N}}+P_{0} / c^{2}\right)$, when configurations with a small $\mathrm{S}$ phase core are unstable and collapse into those with a large $\mathrm{S}$ phase core, will be presented in a separate paper.

The metastable stellar state of the $\mathrm{N}$ phase can be described by central overcompression - the fractional excess of density relative to $\rho_{\mathrm{N}}$,

$\delta \bar{\rho} \equiv \frac{\rho_{\mathrm{c}}-\rho_{\mathrm{N}}}{\rho_{\mathrm{N}}}$

Equivalently, we define a dimensionless central overpressure,

$\delta \bar{P} \equiv \frac{P_{\mathrm{c}}-P_{0}}{P_{0}}$

Let us now consider the timescales and their interplay. First, there is a microscopic nucleation timescale $\tau_{\text {nucl }}(\delta \bar{P})$. The time dependence $\delta \bar{P}(t)$ results from a global evolution of the neutron star and depends on the astrophysical scenario of central compression; it will be characterized by $\tau_{\text {comp }}=P_{0} / \dot{P}_{\mathrm{c}}=1 / \delta \overline{\bar{P}}$. The time needed to compress matter in the center of the star from $P_{0}$ to $P_{\mathrm{c}}$ is, in a linear approximation, equal to $\tau_{\text {comp }} \cdot \delta \bar{P}$. During this time the system remains in a metastable state. In contrast, the functional dependence $\tau_{\text {nucl }}(\delta \bar{P})$ is given by the local dense matter microphysics and is a very sensitive function of $P$, being very large for $P$ close to $P_{0}$ and dropping abruptly above some pressure (see for example, Iida \& Sato 1997). As a result, the condition for the time $t=t_{\text {quake }}$ at which the metastable stellar configuration collapses can be estimated by solving the equation:

$\tau_{\text {nucl }}[\delta \bar{P}(t)]=q \cdot \tau_{\text {comp }} \cdot \delta \bar{P}$

where a small dimensionless coefficient $q$ reflects the very steep character of the function $\tau_{\text {nucl }}(\delta \bar{P})$ and is of the order $10^{-2}-10^{-3}$ (details will be presented in a forthcoming paper). 


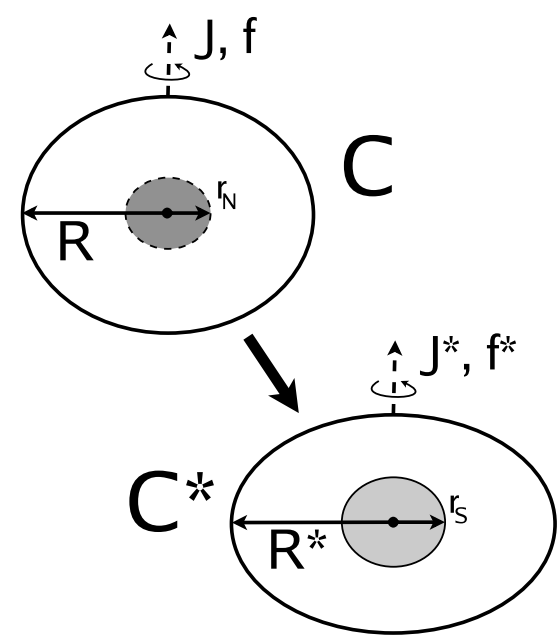

Fig. 2. Transition from a one-phase configuration $C$ with a meta-stable core of radius $r_{\mathrm{N}}$ to a two-phase configuration $C^{\star}$ with $\mathrm{S}$-phase core with a radius $r_{\mathrm{S}}$. By "radius" we mean an equatorial circumferential radius. These two configurations have the same baryon number $A=A^{\star}$ and total angular momentum $J=J^{\star}$.

\section{Calculation of the energy release}

We restrict ourselves to axially symmetric, rigidly rotating neutron stars in hydrostatic equilibrium. In what follows, by "radius" we mean a circumferential radius in an equatorial plane.

We assume that at a central pressure $P_{\mathrm{c}}=P_{\text {nucl }}$ the nucleation of the $\mathrm{S}$ phase in an overcompressed core, of radius $r_{\mathrm{N}}$, of configuration $C$, initiates the phase transition and formation of an $\mathrm{S}$-phase core of radius $r_{\mathrm{s}}$ in a new configuration $C^{*}$, as presented in Fig. 2. Transition to an $\mathrm{S}$ phase at the core boundary, occurring at $r_{\mathrm{S}}$, is associated with a density jump characterized by $\lambda_{\rho}=\rho_{\mathrm{S}} / \rho_{\mathrm{N}}$. Because we are interested in relatively small Sphase cores, we will approximate its EOS by a polytrope with the exponent $\gamma_{\mathrm{S}}$, equal to the adiabatic index of the $S$ phase at $\rho=\rho_{\mathrm{S}}$. Of course, in reality the adiabatic index of the $\mathrm{S}$ phase does depend on the density. However, for small cores the polytropic EOS is an excellent approximation, and in the limit when only leading terms in the $r_{\mathrm{s}}$-expansion are kept, this approximation becomes exact (Bejger et al. 2005).

Having a pair of EOSs, one with and one without a softening by phase transition, the next step is to compare the hydrostatic equilibria of neutron stars corresponding to each of these EOSs. The models that we calculate are rigidly rotating, axisymmetric solutions of Einstein's equations. The numerical computations have been performed by means of a code built on the Lorene library (http: //www . lorene.obspm. fr), with an accuracy of $10^{-6}$ or better measured using general relativistic virial theorems. The neutron-star models can be labeled by the central density $\rho_{\mathrm{c}}$ and rotational frequency $f=\Omega / 2 \pi$. These parameters are natural from the point of view of numerical calculations ( $\rho_{\mathrm{c}}$ and $f$ are input parameters in the numerical code). But we can imagine another parametrization, more useful for other purposes. For example, to study the stability of rotating stars, the better choice is central density, $\rho_{\mathrm{c}}$, and total angular momentum of the star, $J$.

Below $\rho_{\mathrm{N}}$, the EOS for two cases (with and without phase transition) is the same. For the problem of non-rotating stars, considered two decades ago (Haensel et al. 1986; Zdunik et al. 1987), the configuration with $\rho_{\mathrm{c}}=\rho_{\mathrm{N}}$ was denoted by $C_{0}$, and treated as a "reference configuration". Configurations $C_{0}, C$, and $C^{\star}$ are depicted in the $A-P_{\mathrm{c}}$ plane in Fig. 3. The radius,

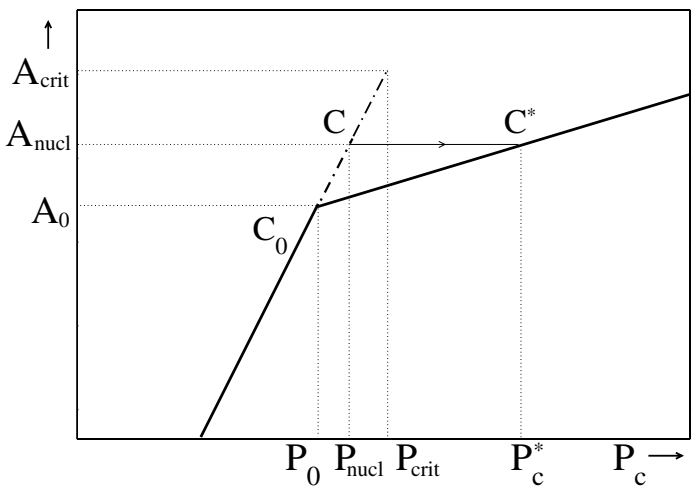

Fig. 3. Total baryon number $A$ of the hydrostatic stellar configuration versus central pressure $P_{\mathrm{c}}$, at fixed stellar angular momentum $J$, for the EOS depicted in Fig. 1. The solid line denotes stable states, dash-dot line - the states that are meta-stable with respect to the $\mathrm{N} \longrightarrow \mathrm{S}$ transition. For a central pressure $P_{\text {nucl }}$ the $\mathrm{S}$-phase nucleates in the supercompressed core of configuration $C$, and this results in a transition $C \longrightarrow C^{*}$ into a stable configuration with a S-phase core and central pressure $P_{\mathrm{c}}^{*}$. Both configurations $C$ and $C^{*}$ have the same baryon number $A$.

gravitational mass, and the total baryon number of $C_{0}$ will be denoted by $R_{0}, M_{0}$, and $A_{0}$. For rotating stars we do not have one "reference configuration" but a set of "reference configurations" which depend on rotation rate $\left\{C_{0}(f)\right\}$ or total angular momentum $\left\{C_{0}(J)\right\}$ of the initial metastable configuration. As discussed in Sect. 2, there also exists a set of configurations resulting from the metastability of the matter in N phase. The maximum central pressure which can be reached in the N-phase star is defined by the value $P_{\mathrm{c}}=P_{\text {nucl }}$. For $P_{\mathrm{c}}>P_{\text {nucl }}$, the phase transition in the center of the star takes place on a timescale much shorter than time of the stellar evolution (accretion or spin down). Thus the "critical line" defined by the condition $P_{\mathrm{c}}=P_{\text {nucl }}$ corresponds to the configurations for which nucleation in the center triggers a collapse of the whole star and a corequake. From the astrophysical point of view, the phase transition in the center followed by a corequake takes place at a point on a "critical line". Consequently, "normalization" of results with respect to parameters of the "reference configuration" is more complicated than in the case of non-rotating stars. There are many possibilities to define the "reference configuration" for a star with a metastable core $\left(P_{\mathrm{c}}>P_{0}\right)$, rotating with frequency $f$ and possessing a total angular momentum $J$ and total baryon number $A$. Such a "reference configuration" should be defined as the configuration with the central pressure equal to $P_{0}$, as shown in Fig. 3. However, we can choose either a reference configuration star with the same $J$ or the same $f$ as that of the collapsing metastable configuration $C$. In fact, to reach the metastable configuration with $P_{\mathrm{c}}>P_{0}$, we have to cross the "reference line" at the point defined by the evolutionary process leading to the instability. Properties of the "critical line" and "reference line" in the space of rotating configurations of slowing down or accreting neutron stars will be analyzed in more detail in a forthcoming paper.

We additionally assume that the transition of the star from a one-phase configuration to the configuration with a small dense core of $\mathrm{S}$ phase, takes place at fixed baryon number $A$ (no matter ejection) and fixed total angular momentum of the star $J$ (radiation loss of $J$ neglected). 


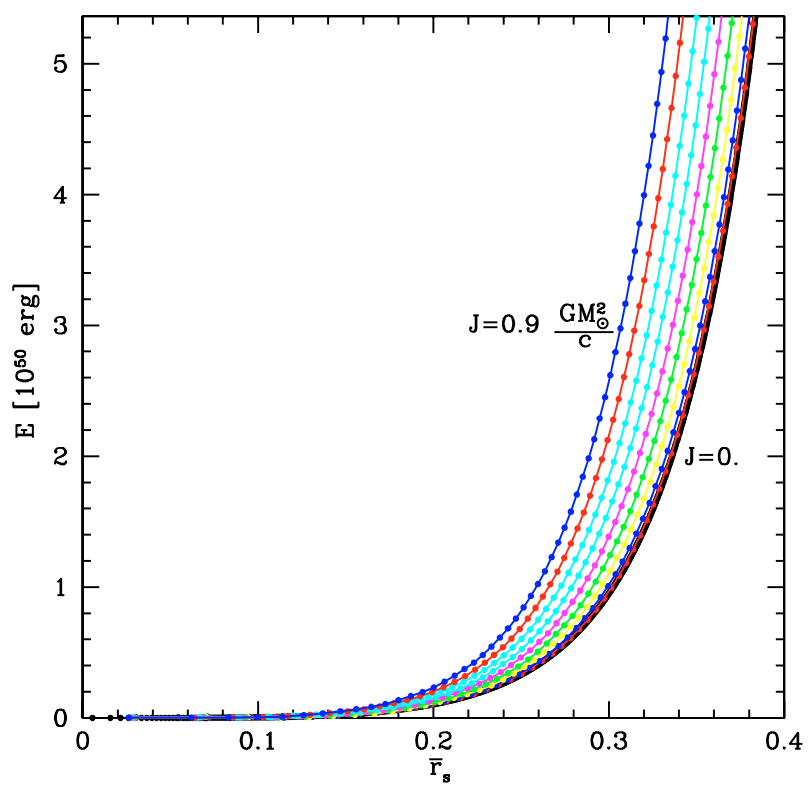

Fig. 4. The energy release due to the corequake of rotating neutron star as a function of the dimensionless equatorial radius of the new phase core, $\bar{r}_{\mathrm{s}}$. Different curves correspond to the different values of total angular momentum of rotating star, fixed along each curve, $J=$ $(0,0.1, \ldots, 0.9) \times G M_{\odot}^{2} / c$, from the bottom curve to the top curve.

The energy release during transition $C(A, f) \longrightarrow C^{\star}\left(A, f^{\star}\right)$ is calculated from the change of the stellar mass-energy during this process,

$\Delta E=\left[M(C)-M\left(C^{\star}\right)\right]_{A, J} c^{2}$.

\subsection{Energy release for polytropic EOSs}

In the present section we simplify the analysis, removing problems connected with numerical precision. To this aim, we will use the polytropic EOSs for the $\mathrm{N}$ and $\mathrm{S}$ phases. The polytropic EOSs not only guarantee a high precision of numerical calculation, but also open the possibility of the exploration of a wide region of the parameter space. A description of the polytropic EOSs and their application to relativistic stars with phase transitions was presented in detail in our previous publications in this series (Bejger et al. 2005; Zdunik et al. 2006).

In Fig. 4 we presented the energy release as a function of $\bar{r}_{\mathrm{s}}=r_{\mathrm{s}} / R(C)$, for several values of the angular momentum of the metastable configurations $C(J)$. The reference configuration is $C$ - the initial configuration of the corequake with a metastable core in the center and central pressure $P_{\mathrm{c}}=P_{\text {nucl }}$. As we see in Fig. 4, the energy release corresponding to a given value of $\bar{r}_{\mathrm{s}}$ depends rather strongly on the rotation rate (here presented for the fixed values of total angular momentum).

In Fig. 5 we presented the energy release as a function of the overpressure of the metastable $\mathrm{N}$ phase in the center of the metastable $\operatorname{star} C(A, J), \delta \bar{P}=P_{\text {nucl }} / P_{0}-1$, for several values of $J$. As we already stressed, the value of $P_{\text {nucl }}$ (or $\delta \bar{P}$ ) can be determined from microscopic considerations, combined with physical conditions prevailing at the star center as well as with their time evolution rate. Having determined $P_{\text {nucl }}$, we can determine the energy release, $\Delta E$, due to the corequake $C(A, J) \longrightarrow C^{\star}(A, J)$, where the metastable one-phase configuration, and the final two-phase configuration, have the same values of the baryon

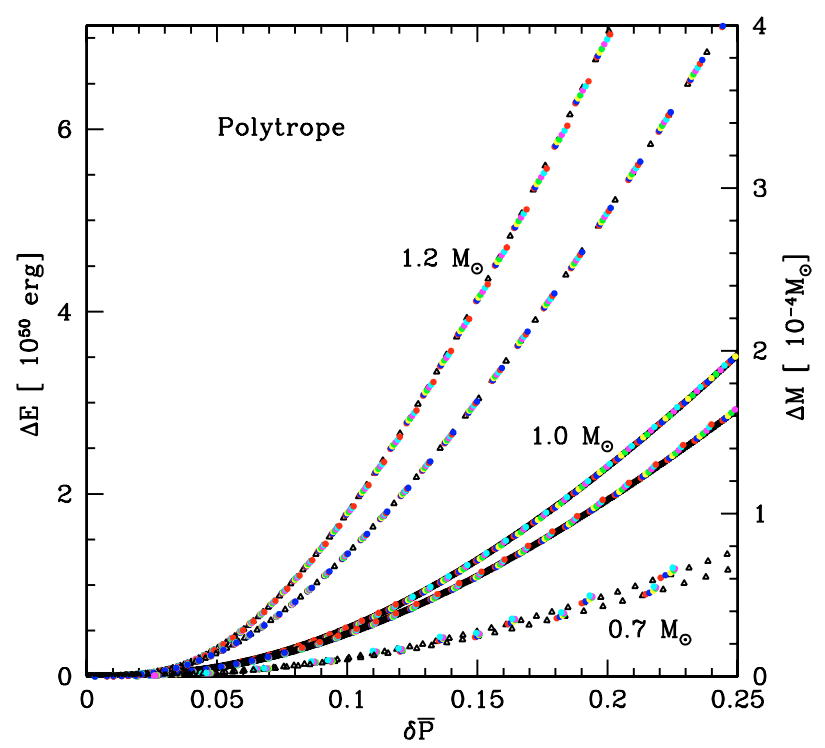

Fig. 5. The energy release due to the mini-collapse of a rotating neutron star as a function of the overpressure $\delta \bar{P}$ of the $\mathrm{N}$ phase of the matter in the center of the star, for the polytropic EOS $(\gamma=2)$ with a first order phase transition located at three different points (pressures $P_{0}$ ) for which the mass of the reference nonrotating configuration is equal to $0.7,1.0,1.2 M_{\odot}$. For each value of $P_{0}$ two models of S phase EOS are considered (one of the examples of the EOS depicted in Fig. 7). They correspond to the $\mathrm{S}$ phase described by the polytropes with $\gamma_{\mathrm{S}}=2.5$ (upper curve) and $\gamma_{\mathrm{S}}=3$ (lower curve). The points of different color correspond to the different values of total angular momentum of the rotating star, $J=(0,0.1, \ldots, 0.9) \times G M_{\odot}^{2} / c$. For a given EOS, results for all rotating configurations can be very well approximated by a single curve, independent of $J$. (This figure is available in color in the electronic form.)

number $A$ and total angular momentum $J$, which are conserved during the transition.

As we see in Fig. 5, the energy release in a (mini)collapse of a rotating star is independent of the rotation rate of the collapsing configuration, and depends exclusively on the degree of metastability of the $\mathrm{N}$ phase at the stellar center (departure of matter from chemical equilibrium), measured by the overpressure $\delta \bar{P}$. In particular, to calculate the energy release associated with a corequake of a rotating neutron star, it is sufficient to know the value of $\Delta E$ for a non-rotating star of the same central overpressure, which can be deduced from expressions derived in Zdunik et al. (1987).

The configurations $C(A, J)$ and $C^{\star}(A, J)$, considered in this section, are really fast rotating ones, not so far from the Keplerian limit. This is visualized in Fig. 6, where we plotted the oblateness of the star and the kinetic to potential energy ratio. And still, in spite of fast rotation and large oblateness, the energy release is the same as in a non-rotating star of the same initial central overpressure.

\subsection{Energy release for realistic EOS}

In the present section we consider a realistic EOS of the $\mathrm{N}$ phase. In order to explore how a realistic rotating neutron star will respond to the appearance of a core of $S$ phase core, we used a recent SLy EOS of Douchin \& Haensel (2001). The SLy EOS describes in a unified way (i.e., starting from a single effective nuclear Hamiltonian) both the crust and the core of a neutron star. In its original version, the SLy EOS assumes that the neu- 

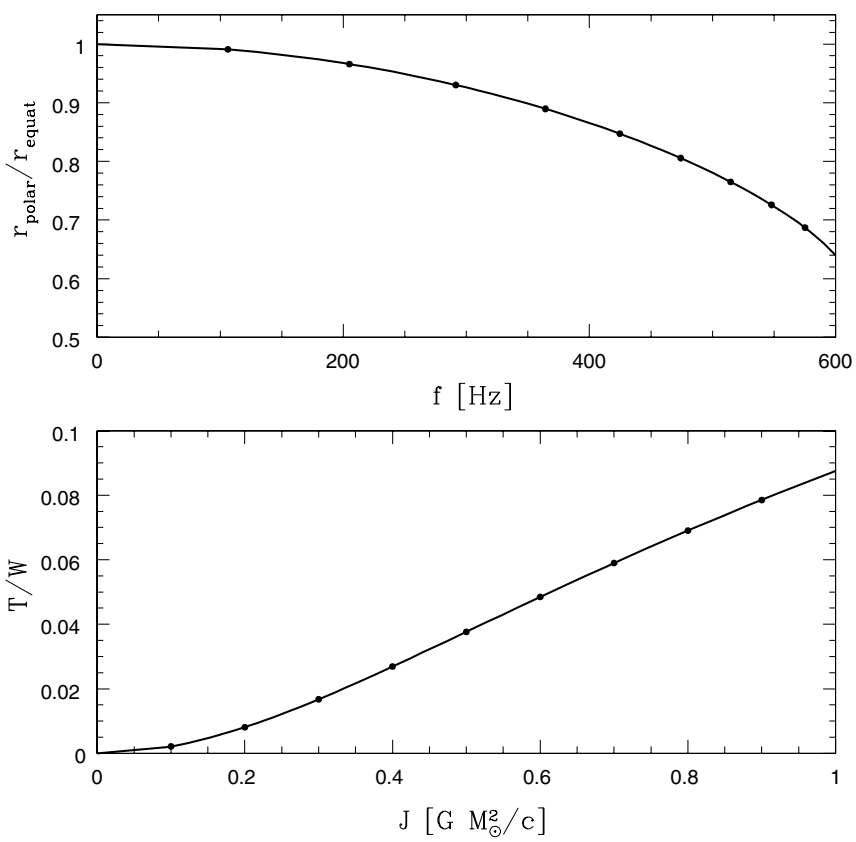

Fig. 6. Top panel: the ratio of polar radial coordinate to the equatorial radial coordinate radius versus frequency of rotation. Bottom panel: the ratio of the kinetic energy, $T$ and the absolute value of the potential energy, $W$ versus stellar angular momentum. Calculations performed for stellar configurations consisting of the $\mathrm{N}$ phase of dense matter, described by the polytropic EOS. Large dots correspond to the values of the total stellar angular momentum, $J=(0.1, \ldots, 0.9) \times G M_{\odot}^{2} / c$, which were used in Figs. 4, 5.

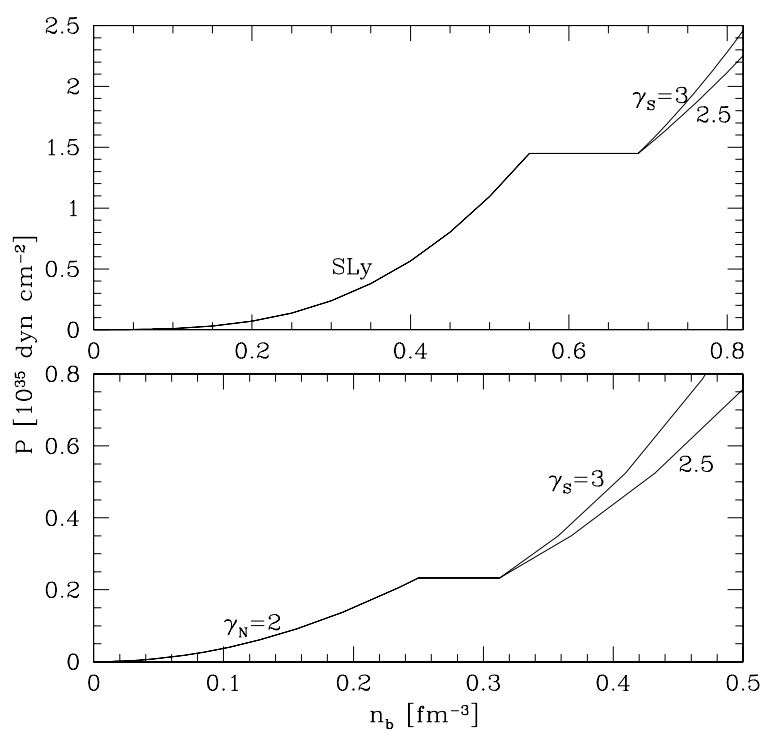

Fig. 7. The EOSs with first order phase transitions, used in the present paper. Upper panel: the SLy EOS for the N phase, with a phase transition to the dense $\mathrm{S}$ phase. We assume $n_{\mathrm{N}}=0.54 \mathrm{fm}^{-3}$ and $\lambda_{n}=n_{\mathrm{S}} / n_{\mathrm{N}}=$ 1.25 , which results in $\lambda_{\rho}=\rho_{\mathrm{S}} / \rho_{\mathrm{N}}=1.28987$. For a further description of the EOS, see the text. Lower panel: polytropic models of the EOS with a first order phase transition. We assume $n_{\mathrm{N}}=0.25 \mathrm{fm}^{-3}$ and $\lambda_{n}=n_{\mathrm{S}} / n_{\mathrm{N}}=1.25$, which results in $\lambda_{\rho}=\rho_{\mathrm{S}} / \rho_{\mathrm{N}}=1.2647$.

tron star core is composed of neutrons, protons, electrons and muons. We introduced a softening by a first order phase transition at $n_{\mathrm{N}}$, as shown in Fig. 7.

We considered two EOSs of the S phase. Both were of polytropic form $P\left(n_{\mathrm{b}}\right)=K_{\mathrm{s}} n_{\mathrm{b}}^{\gamma_{\mathrm{s}}}$, with $\gamma_{\mathrm{s}}=2.5$ and $\gamma_{\mathrm{s}}=3$, respec-

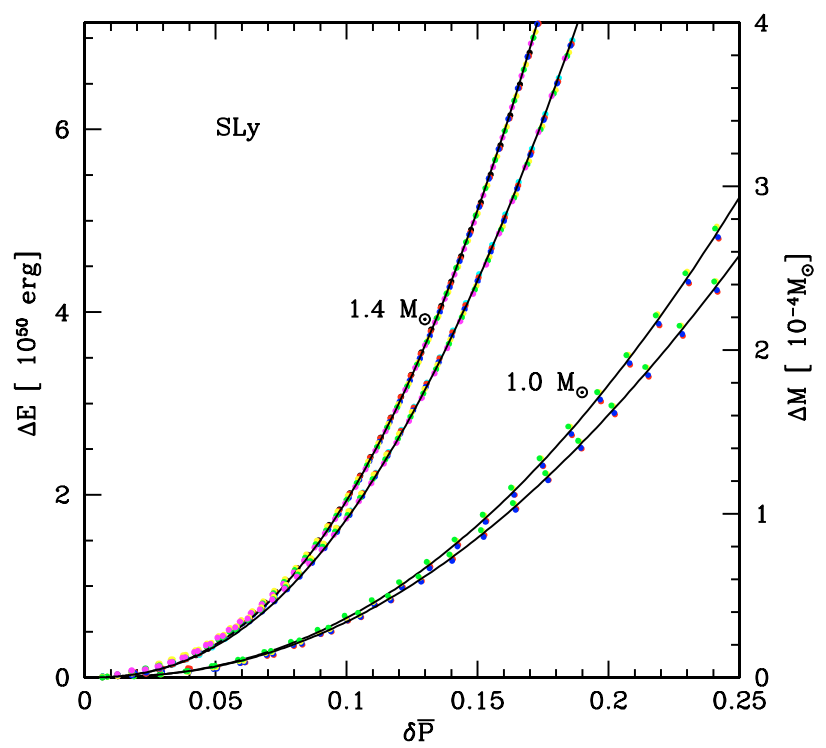

Fig. 8. The energy release due to the mini-collapse of rotating neutron star as a function of the metastability of the normal phase of the matter in the center of the star for the SLy EOS with first order phase transition located at two different points (pressures $P_{0}$ ) for which the mass of the reference nonrotating configuration is equal to $1.0,1.4 M_{\odot}$. For each value of $P_{0}$ two models of the dense core EOS are presented polytropes with $\gamma=2.5$ (upper curve) and $\gamma=3$. The points of different color correspond to the different values of total angular momentum of rotating star. The results for all rotating configurations can be very well approximated by one curve. (This figure is available in color in the electronic form.)

tively. We assume the values of $n_{\mathrm{N}}$ and $n_{\mathrm{S}}=\lambda_{n} n_{\mathrm{N}}$. The values of $n_{\mathrm{N}}$ and $\lambda_{n}$ determine then $P_{0}$. Equality of baryon chemical potentials at $P=P_{0}$ fixes the other constant of the $S$-phase EOS, which is the value of energy per baryon (including rest energy) at zero pressure. In this way, the values of $n_{\mathrm{N}}$ and $\lambda_{n}$, together with $\gamma_{\mathrm{s}}$, fully determine the EOS of the $\mathrm{S}$ phase.

In Fig. 8 we show the energy release due to the $C(A, J) \longrightarrow$ $C^{\star}(A, J)$ transition versus overpressure, for two selected values of $\gamma_{\mathrm{S}}$. As in the case of the polytropic models of the $\mathrm{N}$ phase, all color points lie along the same line. For a given overpressure $\delta \bar{P}$, the energy release does not depend on $J$ of the collapsing metastable configuration, confirming in this way results obtained for the polytropic EOSs. This property is fulfilled very well for a broad range of of stellar angular momentum, $J=(0.1, \ldots, 0.7) \times G M_{\odot}^{2} / c$.

Our numerical results for different equations of state and models of phase transitions presented in Figs. 5 and 8 show that the energy release associated with a phase transition in the core does not depend on the stellar rotation rate for a fixed overpressure at which the phase transition takes place. To estimate the "accuracy" of this conclusion we can calculate the departures of the energy release for the rotating stars from the curve determined for nonrotating configurations. The relative differences are of the order of $1-2 \%$ for $\delta \bar{P}>0.1$ (except for the smallest masses in Figs. 5, $8\left(M=0.7 M_{\odot}\right.$ and $M=1 M_{\odot}$ respectively) where they can be as large as $5 \%$ ). For $\delta \bar{P}<0.1$ the relative differences are larger because the absolute value of $\Delta E$ is very small and the differences are comparable to the numerical accuracy. 


\section{Calculation of the energy release using linear response theory}

Below $\rho_{\mathrm{N}}$, the EOS for two cases (with and without phase transition) is the same. The ambiguity of the definition of $C_{0}$ for rotating stars was discussed in Sect. 3. We can choose a reference configuration star with the same $J$ or the same $f$ as that of collapsing metastable one. To reach the metastable configuration with $P_{\mathrm{c}}>P_{0}$, we have to cross the "reference line" at the point defined by the evolutionary process leading to the instability.

We assume that transition of the star from a one-phase configuration to the configuration with a small dense core built of $\mathrm{S}$ phase takes place at fixed baryon number $A$ (no matter ejection) and fixed total angular momentum of the star $J$ (radiation loss of $J$ neglected). Thus for a fixed baryon number $A$ and fixed $J$, we will calculate the mass-energy difference between $C$ and $C^{\star}$.

We assume that the mass of the S-phase core is much smaller than the total stellar mass. Therefore, from now on we will be able to restrict ourselves to a linear response of neutron star to the appearance of the new dense S-phase core. The calculation is based on expressing the change in the density profile, due to the presence to a small core, as the combination of two independent solutions of the linearly perturbed equations of stellar structure (Haensel et al. 1986; Zdunik et al. 1987). The presence of a denser phase in the core changes the boundary condition at the phase transition pressure $P_{0}$ and allows us to determine the numerical coefficients in the expression for the density profile change. The leading term in the perturbation of the boundary condition at the edge of the new phase results from the mass excess due to the higher density of the new $\mathrm{S}$ phase as compared to the $\mathrm{N}$ one in the supercompressed core.

\subsection{Non-rotating neutron stars}

We use expressions obtained for static case by Haensel et al. (1986), and Zdunik et al. (1987). For a non-rotating configuration of hydrostatic equilibrium, the density jump $\rho_{\mathrm{N}} \rightarrow \rho_{\mathrm{S}}$ leads to the lowest-order expression for the core-mass excess (with respect to the pure N-phase configuration),

$\delta m_{\text {core }}=\frac{4}{3} \pi\left(\rho_{\mathrm{s}}-\rho_{\mathrm{N}}\right) r_{\mathrm{s}}^{3}+O\left(r_{\mathrm{s}}^{5}\right)$.

The radius of the overcompressed $\mathrm{N}$-phase core, $r_{\mathrm{N}}$, is connected to the overpressure that can be achieved by the $\mathrm{N}$ phase of matter by

$\delta \bar{P}=\frac{2 \pi}{3} G x_{\mathrm{N}} \rho_{\mathrm{N}}\left(1+x_{\mathrm{N}}\right)\left(1+3 x_{\mathrm{N}}\right) r_{\mathrm{N}}^{2}$,

where $x_{\mathrm{N}}=P_{0} / \rho_{\mathrm{N}} c^{2}$.

The conservation of the stellar baryon number during the $C \longrightarrow C^{\star}$ transition implies a relation between $r_{\mathrm{N}}$ and $r_{\mathrm{S}}$ in which neglecting terms $r_{\mathrm{N}}^{4}$ and higher, reads

$\left(1+3 x_{\mathrm{N}}\right) r_{\mathrm{N}}^{2}=\left(3-2 \lambda_{\rho}+3 x_{\mathrm{N}}\right) r_{\mathrm{s}}^{2}-\left(1-\lambda_{\rho}\right) a_{\mathrm{N}} r_{\mathrm{s}}^{3}$

where $a_{\mathrm{N}}$ is a numerical coefficient depending on $C_{0}$.

Using then Eqs. (6) and (7), the leading term for the energy release expression $\propto r_{\mathrm{S}}^{5}$ contains a prefactor $\left(\lambda_{\rho}-1\right)\left(3-2 \lambda_{\rho}+3 x_{\mathrm{N}}\right)$, while the next order term $\propto r_{\mathrm{s}}^{6}$ contains a prefactor $\left(\lambda_{\rho}-1\right)^{2}$. The final expression for a normalized energy release reads

$$
\begin{aligned}
\Delta \bar{E} \equiv & \frac{M-M^{*}}{M_{0}} \simeq\left(\lambda_{\rho}-1\right)\left(3-2 \lambda_{\rho}+3 x_{\mathrm{N}}\right) \alpha_{1} \bar{r}_{\mathrm{s}}{ }^{5} \\
& +\left(\lambda_{\rho}-1\right)^{2} \alpha_{2} \bar{r}_{\mathrm{s}}{ }^{6},
\end{aligned}
$$

where $\bar{r}_{\mathrm{s}} \equiv r_{\mathrm{s}} / R_{0}$. The coefficients $\alpha_{1}$ and $\alpha_{2}$ are functionals of the static reference configuration, $C_{0}(J=0)$. Expressing now $\bar{r}_{\mathrm{s}}$ in terms of overpressure, we obtain the energy release in terms of the overpressure,

$\Delta E_{50} \simeq \frac{\left(\lambda_{\rho}-1\right)}{\left(3-2 \lambda_{\rho}+3 x_{\mathrm{N}}\right)^{1.5}} \beta_{1}(\delta \bar{P})^{2.5}$,

where we use standard notation $E_{50} \equiv E / 10^{50} \mathrm{erg}$. The coefficient $\beta_{1}$ depends only on the EOS of the $\mathrm{N}$ phase. More precisely, this coefficient is a functional of the nonrotating reference configuration $C_{0}$ and depends rather weakly on the mass $M_{0}$ of the reference configuration $C_{0}$ for a rather broad range of masses, between $1 M_{\odot}$ and $0.8 M_{\max }$. For EOSs considered in the present paper, numerical calculations give to a good approximation $\beta_{1} \simeq 0.016$.

\subsection{Rotating neutron stars}

The numerical results of Sect. 3 clearly show that for for a given central overpressure of collapsing configuration, $\delta \bar{P}$, the energy release does not depend on $J$ and coincides with that of nonrotating stars. Therefore, the formula (9) of Sect. 4.1 is valid also in the rotating case, provided $\delta \bar{P}$ is sufficiently small. Our calculations show that this formula is quite precise for $\delta \bar{P} \lesssim 0.05$. The prefactor in front of $\beta_{1}$, involving $\lambda_{\rho}$ and $x_{\mathrm{N}}$, is identical to those obtained for the $J=0$ transition in Haensel et al. (1986) and Zdunik et al. (1987). The coefficient $\beta_{1}$ depends only on the EOS of the $\mathrm{N}$ phase. More precisely, this coefficient is a functional of the nonrotating reference configuration $C_{0}$.

\section{Numerical estimates of the energy release}

For practical application, and for small overpressures, $\delta \bar{P}<0.1$, it is convenient to summarize results obtained in Sects. 3.1-3.2 in a formula

$\Delta E_{50}=a_{1}(\delta \bar{P})^{2.5}$

The only dependence of coefficient $a_{1}$ on the phase transition parameter $\lambda_{\rho}$ is via prefactors,

$a_{1}=\frac{\left(\lambda_{\rho}-1\right)}{\left(3-2 \lambda_{\rho}+3 x_{\mathrm{N}}\right)^{1.5}} \beta_{1}$,

which allows for a rapid re-calculation of $a_{1}$ when one changes the value of the density jump.

For the phase transition model considered in Sects. 3.2, the energy accompanying phase transition in a metastable star with central overpressure $\delta \bar{P}=0.1$ is about $2 \times 10^{50} \mathrm{erg}$, and becomes one order of magnitude smaller for $\delta \bar{P}=0.05$.

\section{Discussion and conclusions}

The most important result of the present paper is that the total energy release associated with a first order phase transition at the center of a rotating neutron star depends only on the overpressure at the center of the metastable configuration and is independent of the star rotation rate. This result holds even for fast stellar rotation, when the star shape deviates significantly from sphericity, and $J \simeq 0.9 G M_{\odot}^{2} / c$, and for overpressures as high as $10-20 \%$. This property is of great practical importance because it implies that the calculation of the energy release for 
a given overpressure, requiring very high precision to guarantee $A=A^{\star}$, can be reduced to the case of non-rotating spherically symmetric stars.

To illustrate the practical importance of our result, let us consider the case of overpressure $\delta \bar{P}=0.03$, when the energy release is $2 \times 10^{49} \mathrm{erg}$. For a $1.4 M_{\odot}$ star this constitutes about $10^{-5}$ of the stellar mass-energy. Therefore, to arrive at a meaningful result for the energy of this magnitude, conservation of the total baryon number has to be satisfied at the level of one part in a million, which is next to impossible for a $2 \mathrm{D}$ calculation with a realistic EOS. However, such a precision can be easily reached for spherical stars, provided the EOS is used in a thermodynamically consistent way (see, e.g., Haensel \& Proszynski 1982).

Moreover, we have shown that for overpressures smaller than $5 \%$, the energy release is proportional to power 2.5 of the overpressure, with the coefficient weakly dependent on the mass of collapsing configuration, for stellar masses in the range from $1 M_{\odot}$ to about $0.8 M_{\max }$.

The energy release $\Delta E$ that we calculated is an absolute upper bound on the energies that can be released as a result of a phase transition at the star center. The available channels may include, for example, stellar pulsations, gravitational radiation, heating of stellar interior, and X-ray emission from the neutron star surface. Moreover, the phase transition in a rotating star implies shrinking of stellar radius, decrease of the moment of inertia, and spin-up of rotation. These topics will be considered in our next paper.

The present paper refers to the case of a small core of the superdense phase. Therefore, as the energy release scales as the fifth power of the core radius, the energy release is two-three orders of magnitude smaller than that obtained by, e.g., Berezhiani et al. (2003). The case of phase transitions in rotating neutron stars leading to large cores and energy release $\sim 10^{52}$ erg is now being studied.

Acknowledgements. This work was partially supported by the Polish MEiN grant no. 1P03D.008.27 and by the LEA Astrophysics Poland-France (AstroPF) program. M.B. was partially supported by the Marie Curie Intra-european Fellowship MEIF-CT-2005-023644 within the 6th European Community Framework Programme.

\section{References}

Bejger, M., Haensel, P., \& Zdunik, J. L. 2005, MNRAS, 359, 699 Berezhiani, Z., Bombaci, I., Drago, A., Frontera, F., \& Lavagno, A. 2003, ApJ, 586,1250

Diaz Alonso, J. 1983, A\&A, 125, 287

Douchin, F., \& Haensel, P. 2001, A\&A, 380, 151

Glendenning, N. K. 2000, Compact Stars. Nuclear Physics, Particle Physics, and General Relativity (Berlin: Springer)

Haensel, P., \& Proszynski, M. 1982, ApJ, 258, 306

Haensel, P., Zdunik, J. L., \& Schaeffer, R. 1986, A\&A, 160, 251

Iida, K., \& Sato, K. 1997, Prog. Theor. Phys., 98, 277

Kaempfer, B. 1981, Phys. Lett. B, 101, 366

Schaeffer, R., Haensel, P., \& Zdunik, J. L. 1983, A\&A, 126, 121

Seidov, Z. F. 1971, Sov. Astron.- Astron. Zh., 15, 347

Weber, F. 1999, Pulsars as Astrophysical Laboratories for Nuclear and Particle Physics (Bristol \& Philadelphia: IoP Publishing)

Zdunik, J. L., Haensel, P., \& Schaeffer, R. 1987, A\&A, 172, 95

Zdunik, J. L., Bejger, M., Haensel, P., \& Gourgoulhon, E. 2006, A\&A, 450, 747 